\title{
A SYSTEMATIC REVIEW OF APERTURE SHAPES
}

\author{
A. B. Schultz and T. V. Frazier \\ Department of Physics, University of Nevada, Reno, 89557
}

Abstract: This paper discusses the application of apodization to reflecting telescopes. The diffraction pattern of a telescope, which is the image of a star, can be changed considerably by using different aperture shapes in combination with appropriately shaped occulting masks on the optical axis. Aperture shapes studied were the circular, square, and hexagonal. Polaris (a-UMin) was used as the test system.

\section{APERTURE STOP AND MASK CONSTRUCTION}

A11 apertures and masks were machined from particle board. Each aperture was machined to the outer dimensions of the telescope tube. This method enabled rotation of the stop to be accomplished easily while keeping the aperture centered. The masks were made larger than the secondary mirror and its mount in an effort to symmetrize the aperture.

\section{OBSERVATIONS}

The UNR Observatory is equipped with an Ealing Educator 16"-f/11 cassegrain telescope. All photography was accomplished at the cassegrain focus.

Polaris was chosen for this study because its companion (Polaris B) lies near one of the spikes in the diffraction image formed by the UNR telescope. The position of the companion is 18.3" of arc away from Polaris. All exposures were taken using Kodak spectroscopic film type 103a-E, except for the results using the hexagonal aperture stop and mask which were obtained with film type 103a-F. All photographs were over-exposed to emphasize the diffraction pattern.

\section{PHOTOGRAPHS OF POLARIS}

The photograph of Plate 1.a shows the shadow of the aperture without apodization. The spider which supports the secondary mirror assembly consists of four support arms. The three side lobes appearing in the center pattern are produced by the three corners of the triangular mount for the secondary mirror cell. The photograph of Plate 1.b shows the image of Polaris using the unapodized aperture. Polaris $B$ is almost lost in the diffraction image. We can clearly see the four main diffraction spikes due to the spider. The extraneous smaller spikes surrounding Polaris are caused by tabls holding the primary mirror in place.

The photographs of Plate 2 show the results of circular apodization. Plate 2.a is the shadow of the aperture with a circular aperture stop just large enough to cover the tabs holding the primary mirror in place. The aperture is now 15" across. A circular mask (7 1/4" diameter) is centered over the secondary mirror and its mount. The aperture is now symmetrical. Plate 2.b shows the image of Polaris using this combination of circular aperture and mask. Polaris B is clearly visible. You can also see the four spikes of the diffraction pattern due to the presence of the spider. The image has been cleaned up considerably.

The photographs of Plates 3 and 4 show the results using a square aperture stop and a square mask. Plates $3 . a$ and $4 . a$ present the shadow of the square aperture 
and square mask. Plates 3.b and 4.b show the results using this aperture. Plates 3 and 4 show two different orientations of the aperture and mask with respect to the spider. The central region of the image appears to be square. The prominent spikes in Plate 4.b result from diffraction at the straight edges, while the faint spikes are due to the presence of the spider. In Plate 3.b, the spikes caused by the spider and the straight edges overlap. Hence, the four spikes in Plate 3.b are more pronounced than the four major spikes in Plate 4.b. In Plate 3.b, Polaris B is partially obscured by a spike, while Polaris B is easily observed in Plate 4.b.

The photographs of Plates 5 and 6 show the effects of hexagonal apodization on the image. Plates $5 . a$ and 6 . a show the shadow of the hexagonal aperture and hexagonal mask in two different orientations with respect to the spider. Plates 5.b and 6.b present the results using this aperture. The central region of the image appears to be hexagonal. The two prominent spikes in Plate 5.b and 6.b result from the overlap of spikes formed by diffraction at the straight edges and the spikes form by the spider. Polaris B is partially obscured by a spike in Plate 5.b, while it is easily observed in Plate 6.b.

\section{REMARKS}

The three shapes of the aperture studied (circular, square, and hexagonal) present a mixture of improvements in the diffraction image of a reflecting telescope. The circular aperture in combination with a circular mask showed the least improvement in observing double stars, while the square aperture in combination with a square mask seems to show the most improvement in observing double stars. The hexagonal aperture in combination with a hexagonal mask has shown intermediate results between those of the square and circular apertures.

\section{REFERENCES}

1. Everhart, E. and Kantorski, J. W., 1959, Astron. J., 64, 455.

2. Jackson, J. D., 1975, Classical Electrodynamics, John Wiley \& Sons, New York, 427.

3. Born, M. and Wolf, E., 1975, Principles of Optics, Pergamon Press, New York, 387.

4. Jacquinot, P. and Roizen-Dossier, B., 1964, Progress in Optics, Vol. 3 (ed. E. Wolf; North-Holland Publishing Co., Amsterdam), 29.

5. van Albada, G. B., 1958, Contrib. Bosscha Obs., 1958a, No. 6, 3. 


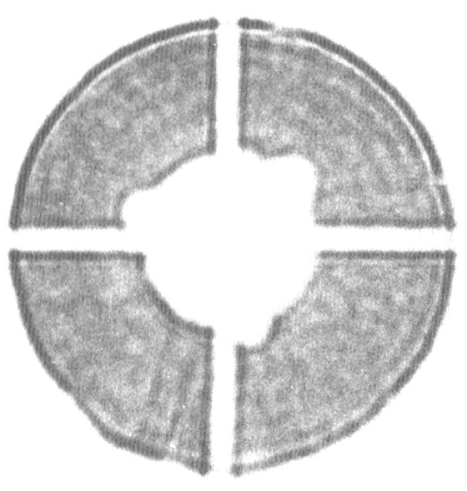

Plate 1.a

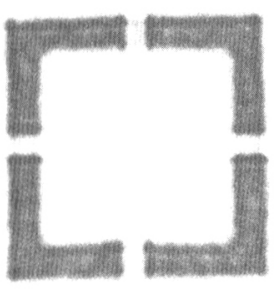

Plate 3.a

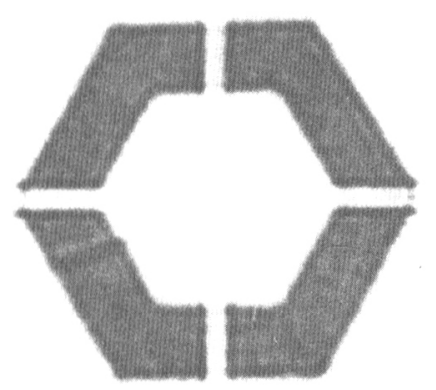

Plate 5.a

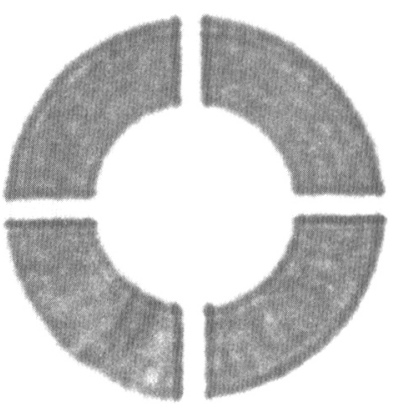

Plate 1.b

Plate 2.a

Plate 2.b

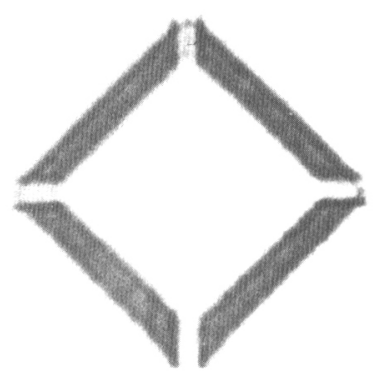

Plate $4 . b$
Plate $4 . \mathrm{a}$

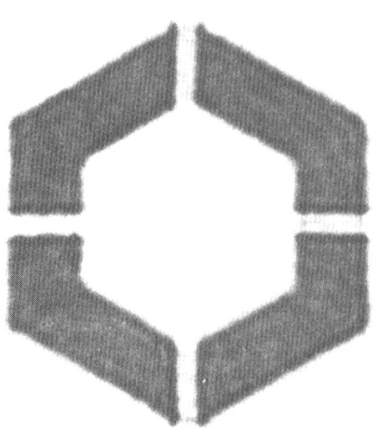

Plate 6.a

端

Plate 3.b

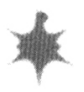

Plate 5.b 


\section{DISCUSSION}

EVANS: I seem to remember at the end of World War II in a French journal a prescription for the removal of diffraction spikes by putting shaped pieces on the supporting pins of the secondary mirror spider.

HOAG: That was the Couder screen. The difficulty is that the image is much fuzzier, and I think the resolution is not improved.

STRAND: A similar technique, using a hexagonal aperture combined with a grating, has been used successfully by I. Lindenblad at the U. S. Naval Observatory to measure the relative positions of the components of Sirius.

SCHULTZ: Yes, he had wires across as a diffraction grating; he did not use a clear aperture.

BAUM: Diffraction can more effectively be removed by attaching a Lyot-type coronagraphic optical system at the focus of the telescope. A transfer lens relays the star image to the detector, and a pupil is formed (with the aid of a field lens) inside the transfer lens. By use of a suitable mask at the pupil image, diffracting portions of the telescope aperture are deleted from the beam before it reaches the detector.

WORLEY: Apodizing diaphragms were used by visual observers such as F. J. W. Hersche 1 and $W$. R. Dawes 150 years ago. 